\title{
Object-oriented classification of multi-resolution images for the extraction of narrow linear forest disturbance
}

\author{
YUHONG HE*†, STEVEN E. FRANKLIN†, XULIN GUO§ \\ and GORDON B. STENHOUSEII \\ $\dagger$ Department of Geography, University of Toronto Mississauga, \\ Mississauga, ON, Canada \\ $\Varangle$ Department of Geography, Trent University, Peterborough, ON, Canada \\ $\S$ Department of Geography and Planning, University of Saskatchewan, \\ Saskatoon, SK, Canada \\ IIAlberta Sustainable Resource Development, Hinton, AB, Canada
}

(Received 15 March 2010; in final form 22 June 2010)

\begin{abstract}
Narrow linear forest disturbances (e.g. seismic cut lines) have been found to have significant effects on wildlife habitat and biodiversity (e.g. species richness and abundance). A great deal of seismic cut lines is created in oil and gas exploration in natural forest areas every year. Accurate mapping of seismic cut lines can therefore contribute to a better understanding of wildlife habitat and biodiversity. However, previous studies have indicated that seismic cut lines were fairly difficult to detect and map even with the available high-spatial resolution imagery (e.g. SPOT 5). Recent progress in feature segmentation and extraction software, such as Definiens Developer 7.0, has enhanced remote sensing capabilities, with the promise of being able to automate tasks. This study investigated the imagery (high resolution or very high resolution) best suited for extracting seismic cut lines using a set of rules and multi-resolution object-oriented classification methods. The data used include SPOT 5 and QuickBird multispectral images and existing GIS databases within one bear management area (BMA) in the eastern slopes of the Rocky Mountains in Alberta. Results indicated that among the available algorithms in the Definiens Developer 7.0 package, the Lee sigma algorithm was capable of highlighting cut lines using the near-infrared (NIR) band of the SPOT 5 image and the QuickBird image. The multi-resolution segmentation was able to segment fresh cut lines when giving higher weight to the Lee sigma edge extraction layer, and the nearest-neighbour object-oriented classifier was able to classify linear features, but with noise. Classification accuracy increased following the post-classification refinement processing. The accuracy assessment indicated that, in the case of delineating fresh cut lines, the higher resolution QuickBird image performed better than SPOT 5. However, neither the QuickBird image nor the SPOT 5 image could accurately delineate relatively old cut lines.
\end{abstract}

\section{Introduction}

Developments associated with oil and gas exploration have expanded rapidly in the natural forest areas, such as the Foothills Region of Alberta, Canada (Schneider et al. 2003, Linke et al. 2005). To locate oil and natural gas deposits, seismic survey is used, 40

*Corresponding author. Email: yuhong.he@utoronto.ca 
and as a result, a series of parallel lines of vegetation are cleared during seismic exploration, leaving long, narrow cut lines, called seismic cut lines. Recent work has indicated that forest cut lines may have a significant effect on important wildlife-habitat relationships (Bayne et al. 2005, Linke et al. 2008). For example, when a network of 5-10 m wide seismic cut lines is created for oil and gas exploration, contiguous landscape patches are dissected and thereby the isolation of patches in this landscape is increased (Linke et al. 2005). Forest cut lines are often used as recreational pathways for off-road vehicles and, therefore, may persist on the landscape (Revel et al. 1984). Complete recovery could take more than 100 years, even in the absence of any human activity after oil and gas exploration (Linke et al. 2008). Persistent use of and new exploration activities are expected to increase forest cut line densities significantly in the future (Schneider et al. 2003).

A reliable and up-to-date cut line map is essential to fully understand the current state of the study habitat and to ensure that adequate attention is paid to the effect of this disturbance on native species. The cut line map is also required for a wide variety of forest planning, management and modelling activities. However, the seismic cut lines are too small to be reliably detected and mapped with coarse- and mediumspatial resolution imagery. In recent years, the increasing availability of high-spatial resolution imagery from several satellite platforms with resolutions better than $10 \mathrm{~m}$ provides greater opportunities for the visual recognition of subtle features. A substantive body of literature has explored the feasibility of the high resolution images for identifying linear features (e.g. Gruen and Li 1995, Katartzis et al. 2001, Hellwich et al. 2002, Kim et al. 2004, Péteri et al. 2004, Quackenbush 2004, Inglada 2007). At the same time, the image-processing capabilities for the detection and extraction of linear features have also improved. Given the significant impacts of the current 65 human disturbances in the Alberta forest areas, it is urgent to assess what new remotely sensed data and processing methods may have potential for detecting and extracting forest cut lines.

This work was built upon the results obtained by a previous study (He et al. 2009), in which a framework was proposed to delineate several disturbance features (cut 70 blocks, roads, cut lines, etc.). We found from the previous work that among all disturbance features, spectral similarities and spatial heterogeneity make it impossible to completely extract cut lines using the framework.

The previous study also indicated that parts of cut lines were not extracted because it is difficult to define a cut line model (i.e. a set of rules based on image spectral and textural characteristics to extract cut lines), which could fit all the different types of cut lines in the image. A typical type of cut lines is 'frequently used' or 'recent' cut lines, which were covered by gravel and had a very bright tone or high reflectance in the image. A model can be defined for these types of cut lines when extracting the features. The second type is 'rarely used' or 'old' cut lines, which were covered by 80 replanted vegetation and had totally different reflectance features from the first type of cut lines. The second type is not of interest in this study because it has been replanted and has less effect on native species. A large amount of cut lines belong to the third type, which is between the first two types - where the degree of contrast between them and their surroundings is various. Even for an individual cut line, the contrast could be low in some parts and high in others. Consequently, it is a challenge to find a model to fit the third type for automatic feature extraction.

Recent advances in feature segmentation and extraction software, such as Definiens Developer 7.0 (Munich, Germany, available at www.definiens.com), have 
enhanced remote sensing capabilities with the promise of being able to automate tasks 90 that were once only possible using time-consuming on-screen digitizing. Although this latest generation of the software offers greater functionality, it is not a panacea for automatic and accurate extraction of features. Using high-spatial resolution image (SPOT 5) and very high spatial resolution image (QuickBird), this study sought to develop the rules and work flow to extract first and third types of cut lines using 95 Definiens Developer 7.0, based on the hypothesis that the newly developed Definiens functions can enhance feature edge, and are able to automate tasks that were once only possible using time-consuming on-screen digitizing and enhancement technique.

\section{Study area and methodology}

\subsection{Study area}

The study is conducted in a bear management area (BMA) of the Foothills Model Forest, located on the eastern slopes of the Rocky Mountains within the foothills of Alberta, Canada (figure 1). This area represents prime grizzly bear habitat but is also an area of significant human use and change due to forestry practices (e.g. harvesting, plantations), and oil and gas exploration and development. The dominant distur- 105 bance features include all-terrain vehicle (ATV) trails, seismic cut lines, cut blocks and
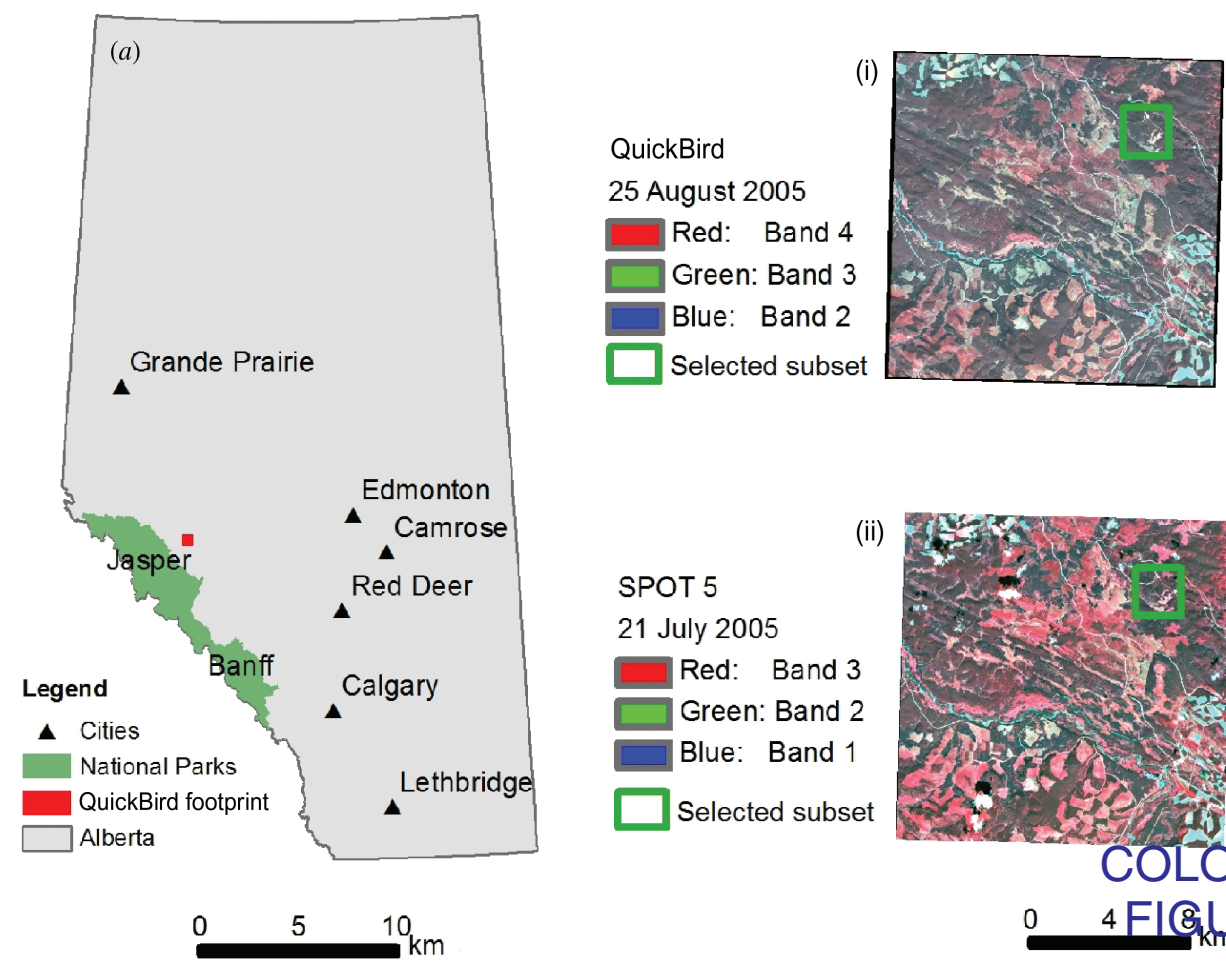

SPOT 5

21 July 2005

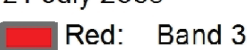
Green: Band 2 Blue: Band 1 Selected subset

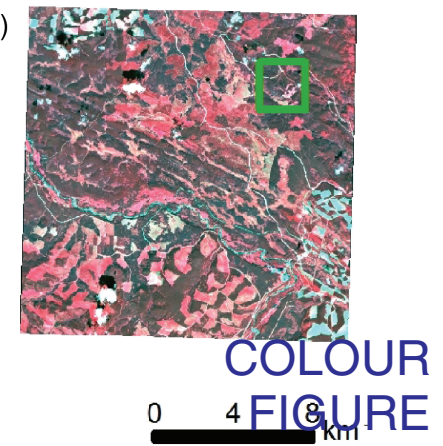

Figure 1. The red square point on the left side showing the study area, located at the foothill region of Alberta (a), Canada; and the images on the right side showing the (i) QuickBird and (ii) SPOT 5 multispectral images in the standard false colour. The green square in the two images indicated the selected subset for discussion in the result section. 
forest roads (figure 1). This boreal forest environment contains pure and mixed forest stands of white spruce (Picea glauca), lodgepole pine (Pinus contorta), trembling aspen (Populus tremuloides) and considerable areas of herbaceous meadows, woody shrubs and treed and non-treed wetlands.

\subsection{Data and preprocessing}

A SPOT-5 10-m multispectral image was acquired on 21 July 2005, and a QuickBird 2.4-m multispectral image was acquired on 25 August 2005 (figure 1). Both satellite images were geometrically corrected using PCI Geomatica 10 (PCI Geomatics, Toronto, Canada). An accuracy of 0.3 pixel root mean square (RMS) or better 115 (representing $\sim 6 \mathrm{~m}$ or less error on the earth's surface) was ensured in the geometric correction process based on 30 ground control points. Topographic distortions were corrected using a 25-m digital elevation model (DEM), originally produced from $1: 20,000$ black and white stereopairs of metric aerial photography (Franklin et al. 2002). Atmospheric and radiometric corrections were completed using the ATCOR-2 algorithm in PCI Geomatica V10. ATCOR-2 uses a sensor-specific atmospheric database of look-up tables containing the results of pre-calculated radiative transfer calculations to remove the effects of atmosphere from the spectral values of the data, as well as correcting the influences of solar illumination and sensor-viewing geometry. Finally, the corrected SPOT image was clipped using the QuickBird footprint, approximating $269 \mathrm{~km}^{2}(16.4 \mathrm{~km} \times 16.4 \mathrm{~km})$, as shown in figure 1 . The time period for the reference data (i.e. the cut lines in the existing GIS database for the study area) was from 1995 to 2005. In such a time period, the cut lines in the reference file covered all cut line types mentioned above.

\subsection{Methods}

A commonly used feature edge enhancement technique - Lee's sigma edge enhancement - is available in Definiens Professional 7.0 package. This edge enhancement approach has been demonstrated to be powerful in smoothing although still preserving and even enhancing image edges (Lee 1983, Rieger et al. 1999). Therefore, we adopted this technique to the input images to suppress noise although enhancing cut line edges. Specifically, the central pixel values were replaced with the mean of a $3 \times 3$ kernel, if the values of the neighbouring pixels are exceeded $2 \sigma$ of the central pixel values, where $\sigma$ is the sigma parameter found by trial and error for the entire image (Definiens 2007).

A multi-resolution image segmentation was performed using the software Definiens Professional 7.0 to create cut line primitives using satellite bands and edge enhancement results. The process uses an iterative, bottom-up, two region-merging algorithm starting with individual pixels. Small objects are merged into larger ones, and the size of the object is controlled by a threshold defined by the scale, shape and compactness parameters specified by the user in a local optimization procedure, which minimized the weighted heterogeneity of each resulting image object. The basic principle for defining the appropriate thresholds for SPOT and QuickBird imagery was that the segmented objects should represent part of cut lines. For this study area, the most suitable parameters to run segmentation for SPOT 5 were found to be scale $=5$, shape $=0.5$ and compactness $=0.5$; and for QuickBird were found to be scale $=10$, shape $=0.5$ and 150 compactness $=0.5$. 
The spectral bands were used for the classification. The feature view was run to investigate the rules that would help separate cut lines from other features. Rules included both spectral and textural information. For example, we found that the most important function to extract cut lines in this study was a grey-level difference vector (GLDV) contrast function computed from near-infrared (NIR) band, and its threshold for SPOT 5 is between 6 and 30. This rule resulted in a classification, in which linear targets were detected but not all cut lines. We did not provide readers with the rest-specific spectra and textural criteria as they are site-/time-specific. Our set of parameters may not work in a different study area with different types of cut lines.

The classification was refined, resulting in a more accurate extraction of cut lines. Several methods were considered. The first was developing a contextual classification rule whereby if a line segment belonged to a cut line, it was assumed that there were probably more line segments in the neighbourhood. This can be checked using the contextual parameters such as existence of, distance to or relative border to. In this way, roads, tails or river bands can be partially separated. The second method involved shape indices, such as width, length/width and shape index to further separate cut lines from forest roads. For example, a rule of width $\leq 20 \mathrm{~m}$ can be used to remove forest roads while keeping cut lines in the output. In addition, small loose segments that are probably not cut line segments can be deleted using the shape and size parameters in Definiens 7.0. The third method utilized the 'merge class' functionality. This was used to connect separate line segments.

\subsection{Validation}

The evaluation criteria (errors of commission and errors of omission) were used to evaluate the accuracy of feature mapping work in this analysis. It is assumed that only linear features within a buffer of a certain width (usually the average width of the features), for example, two SPOT-5 multispectral pixels on both sides of a cut line, are correct. The extracted features which are inside the buffer of the given reference features were considered a successful match. In this study, a buffer of $10 \mathrm{~m}$ (the maximum width of cut lines) on both sides of the cut lines in the reference map was used. The equations for the errors of commission and the errors of omission can be found from He et al. (2009). Errors of omission is defined as the percentage of the reference feature data (the cut lines in the existing shape file), which is not explained by the extracted feature data, that is, the part of the reference feature data which does not lie within the buffer around the extracted data. Errors of commission represent 185 the percentage of incorrectly extracted feature data, that is, the part of the extracted data which does not lie within the buffer around the reference data.

The steps (2.3-2.4) were applied to entire QuickBird and SPOT images displayed in figure 1. Therefore, the quantitative results in the next section are for the full scene. However, only a subset of images (see the boundary of the selected subset in figure 1) 190 and their corresponding products were provided in the result section so that the resultant maps can be viewed visually.

\section{Results and discussion}

Figure 2(a) and $(b)$ showed the subset of raw images in the standard false colour composite, and figure $2(c)$ and $(d)$ showed the best Lee sigma enhancement results (from NIR bands). From the subset of raw images, we can see nine cut lines, two parts of highway, four cut blocks and some rivers or streams. A strong contrast between cut 

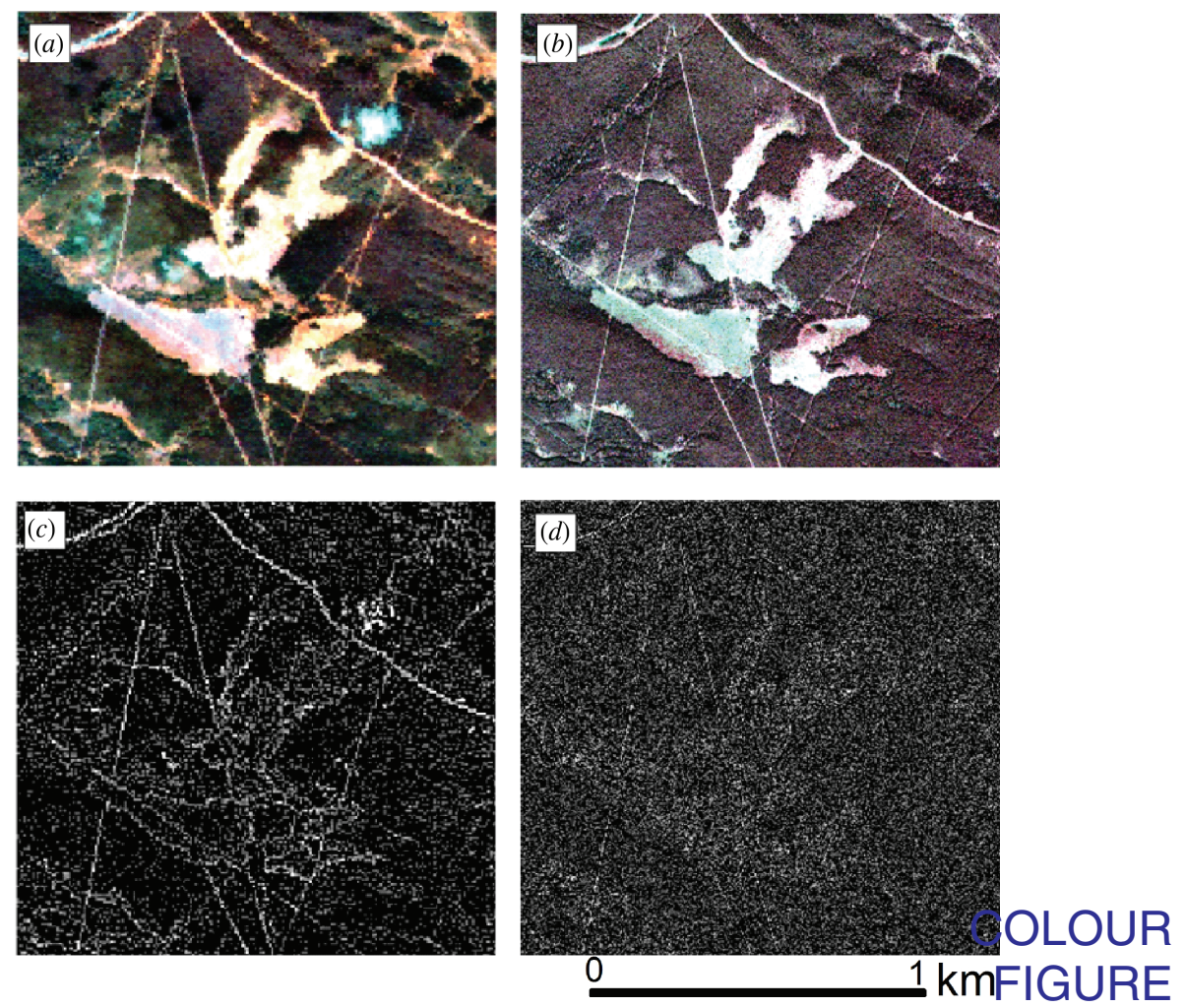

Figure 2. Lee sigma enhancement results for the subset of SPOT 5 and QuickBird multispectral images, $(a)$ is a portion of SPOT 5 image in the standard false colour composite, $(b)$ is a portion of QuickBird image in the standard false colour composite, $(c)$ is the Lee sigma enhancement result for NIR band of the SPOT 5 image and $(d)$ is the Lee sigma enhancement result for NIR band of the QuickBird image.

lines and their surroundings was shown in the SPOT 5 enhancement map. However, some light grey lines or curved lines in the SPOT 5 enhancement map indicated potential problematic areas, that is, cut blocks boundary, highway and river bands. 200 Comparing with SPOT 5 enhancement map, the lower contrast was seen in the QuickBird enhancement map. This result indicated that the higher resolution imagery introduced the high complexity of the image content, especially when the study area is heterogeneous.

Figure 3 showed classification results, post-classification refining process results for 205 the subset of SPOT 5 and QuickBird images and the corresponding reference map. As can be seen, three 'frequently used' or 'recent' cut lines (north-east direction) were classified well, although the QuickBird cut lines were more clearly delineated. However, the third and the most variable type of cut line was not delineated clearly in either of the two images, and some other features such as the highway, cut block 210 boundary and river bands were mistakenly classified as cut lines. Compared with SPOT classification results, the QuickBird classification map had slightly more noisy objects generated during the processing steps; however, these were removed from both maps after the post-classification refining process, based on the width, length and length/width. Although the 'merge class' function was applied and worked in 215 

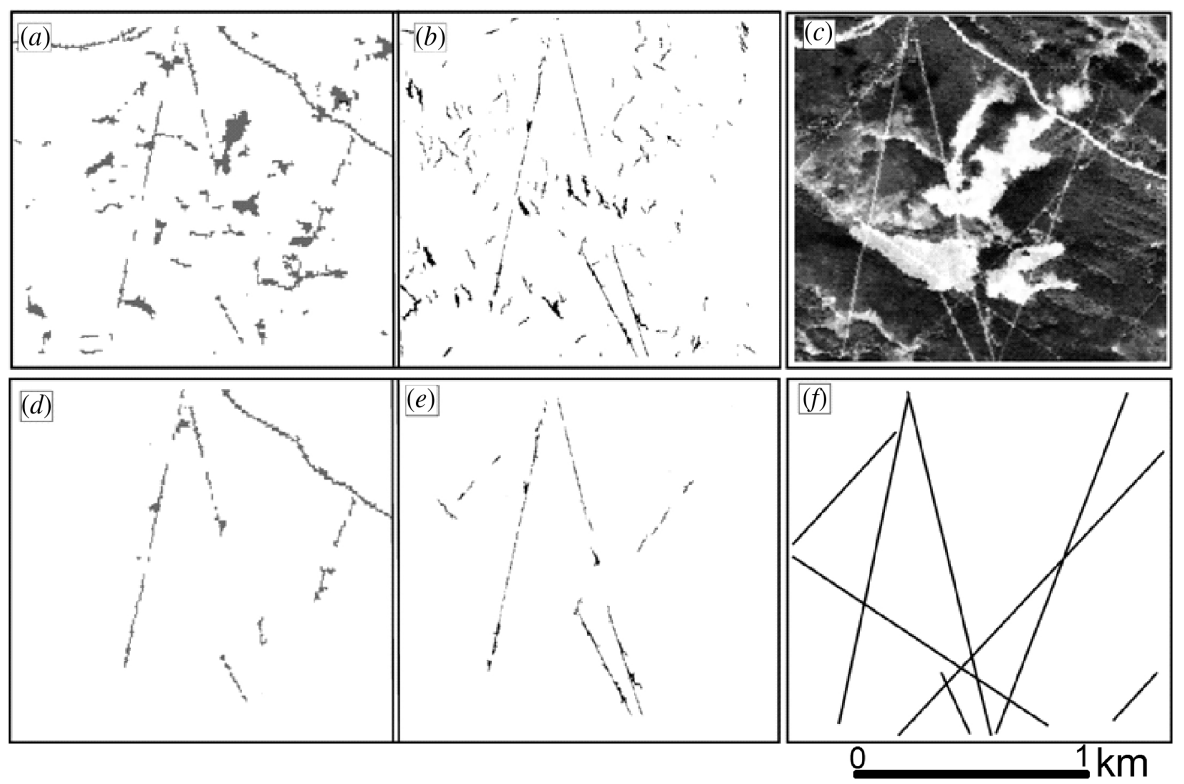

Figure 3. Classification results, post-classification refining process results for the subset of SPOT 5 and QuickBird images and the corresponding reference map. $(a)$ and $(b)$ are classification results for SPOT and QuickBird multispectral images, respectively. $(c)$ is the reference SPOT 5 image. $(d)$ and $(e)$ are post-classification refining results for the SPOT 5 and QuickBird, respectively. $(f)$ is the reference GIS cut line layer.

some cases, other separate cut line segments were still unable to be merged whereas conversely some valuable cut line features were eliminated from the both maps. Overall, the QuickBird-classified cut lines were better in terms of completeness and accuracy.

The resulting errors of commission were 18 and 32\% for QuickBird and SPOT extracted cut lines, respectively. The higher errors of commission for SPOT-extracted map were due to the presence of the highway, which was cutting through the top right of the image from north-west to south-east. The width of the extracted highway in the SPOT classification map was close to the width of cut lines; therefore, this noisy feature was not able to be removed. The QuickBird classification map accurately recognized the highway as a noisy object, thereby removing it entirely which yielded a lower error of commission. The errors of omission were 52 and 63\% for QuickBirdand SPOT-extracted cut lines, respectively. The high errors of omission were attributed to the second or third type of cut lines, which were covered or partially covered by replanted vegetation and had totally different reflectance features from the recent 230 cut lines. Therefore, the high errors of omission may not be a great concern as we can accurately detect the first type of cut lines, which is a way more important factor in the wildlife-habitat studies.

\section{Conclusions}

The results of this analysis have important implications regarding the use of remote sensing to delineate cut lines in a geographically large area. Overall, higher resolution imagery such as QuickBird performed better than high resolution satellites such as 
SPOT 5 in terms of completeness of cut line delineation, accurate removal of noisy objects and lower errors of commission and errors of omission. QuickBird imagery is also more visually appealing for detecting recent cut lines. To extract replanted or relatively old cut lines, neither 2.5 nor $10 \mathrm{~m}$ pixel resolution is sufficient, as the contrast between cut lines and the surrounding natural environment is too low. The problems with higher resolution imagery for extracting cut lines at the landscape scale lies in the difficulty and cost of gathering a comprehensive set of imagery for the entire area of interest and the inherent difficulties of processing complex images - especially when the study area is heterogeneous. While the use of high resolution imagery such as QuickBird is effective, it is also time-consuming and costly to extract cut lines even in a small geographic area, let alone for an entire region (i.e. foothill region or Alberta forest area). The Definiens software provides an attractive alternative to extracting cut lines, but at this time no tools are sophisticated enough to provide a completely automated extraction for all types of forest cut lines.

Further studies are required to extend research to testing the suitability of emerging methods and exploring other remote sensing data types (e.g. multi-angle remote sensing data or airborne laser scanning) for detecting such cut lines. In addition, research could also focus on reducing the omission errors, even at the expense of an 255 increased commission error, as not detecting the features seems to be worse than detecting too many.

\section{Acknowledgements}

This study was supported by the Natural Sciences and Engineering Research Council of Canada, the Alberta Innovation and Science Fund and the many partners of the Foothills Model Forest Grizzly Bear Research Program. We greatly acknowledge the valuable comments and suggestions from the anonymous reviewers for the dramatic improvement in the manuscript.

\section{References}

Bayne, E.M., Van Wilgenburg, S., Boutin, S. and Hobson, K.A., 2005, Modeling and field- 265 testing of ovenbird (Seiurus aurocapillus) responses to boreal forest dissection by energy sector development at multiple spatial scales. Landscape Ecology, 20, pp. 203-216.

Definiens, A.G., 2007, Definiens Developer 7 Reference Book (Munich, Germany: Definiens AG). Franklin, S.E., Peddle, D.R., DechKa, J.A. and Stenhouse, G.B., 2002, Evidential reasoning with Landsat TM, DEM and GIS data for landcover classification in support of grizzly 270 bear habitat mapping. International Journal of Remote Sensing, 23, pp. 4633-4652.

Gruen, A. and Li, H., 1995, Road extraction from aerial and satellite images by dynamic programming. ISPRS Journal of Photogrammetry and Remote Sensing, 50, pp. 11-20.

He, Y., Franklin, S.E., Guo, X. and Stenhouse, G.B., 2009, Narrow-linear and small-area forest disturbance detection and mapping from high spatial resolution imagery. Journal 275 of Applied Remote Sensing, 3, 033570, doi: 10.1117/1.3283905.

Hellwich, O., Laptev, I. and Mayer, H., 2002, Extraction of linear objects from interferometric SAR data. International Journal of Remote Sensing, 23, pp. 461-475.

INGLADA, J., 2007, Automatic recognition of man-made objects in high resolution optical remote sensing images by SVM classification of geometric image features. JSPRS 280 Journal of Photogrammetry \& Remote Sensing, 62, pp. 236-248.

Katartzis, A., Sahli, H., Pizurica, V. and Cornelis, J., 2001, A modelbased approach to automatic extraction of linear features from airborne images. IEEE Transactions on Geoscience and Remote Sensing, 39, pp. 2073-2079. 
Kim, T., Park, S., Kim, M., Jeong, S. and Kim, K., 2004, Tracking road centerlines from high 285 resolution remote sensing images by least squares correlation matching. Photogrammetric Engineering \& Remote Sensing, 70, pp. 1417-1422.

LEE, J.S., 1983, Digital image smoothing and the sigma filter. Computer Vision, Graphics and Image Processing, 24, pp. 255-269.

Linke, J., Franklin, S.E., Huettmann, F. and Stenhouse, G.B., 2005, Changing landscape 290 metrics and grizzly bear landscape use in Alberta. Landscape Ecology, 20, pp. 811-826.

Linke, J., Franklin, S.E., Huettmann, F. and Stenhouse, G.B., 2008, Effects of cut line density and land-cover heterogeneity on landscape metrics in western Alberta. Canadian Journal of Remote Sensing, 34, pp. 390-404.

Péteri, R., Couloigner, I. and Ranchin, T., 2004, Quantitatively assessing roads extracted 295 from high-resolution imagery. Photogrammetric Engineering \& Remote Sensing, 70, pp. 1449-1456.

QuAcKenbush, L.J., 2004, A review of techniques for extracting linear features from imagery. Photogrammetric Engineering \& Remote Sensing, 70, pp. 1384-1392.

Revel, R.D., Dougherty, T.D. and Downing, D.J., 1984, Forest Growth and Revegetation 300 along Seismic Lines (Calgary, AB: The University of Calgary Press).

Rieger, W., Kerschner, M., Reiter, T. and Rottensteiner, F., 1999, Roads and buildings from laser scanner data within a forest enterprise. In International Archives of Photogrammetry and Remote Sensing XXXII/3-W14, pp. 185-191, LaJolla, CA.

Schneider, R.R., Stelfox, J.B., Boutin, S. and Wasel, S., 2003, Managing the cumulative 305 impacts of land uses in the Western Canadian Sedimentary Basin: a modeling approach. Conservation Ecology, 7, p. 8. 